\title{
Expressions for the Celestial Intermediate Pole and Celestial Ephemeris Origin consistent with the IAU 2000A precession-nutation model
}

\author{
N. Capitaine ${ }^{1}$, J. Chapront ${ }^{1}$, S. Lambert ${ }^{1}$, and P. T. Wallace ${ }^{2}$ \\ ${ }^{1}$ Observatoire de Paris, SYRTE/UMR8630-CNRS, 61 avenue de l'Observatoire, 75014 Paris, France \\ ${ }^{2}$ H.M. Nautical Almanac Office, Space Science and Technology Department, CLRC / Rutherford Appleton Laboratory, UK
}

Received 18 July 2002 / Accepted 9 January 2003

\begin{abstract}
Expressions for the position of the Celestial Intermediate Pole (CIP) and the Celestial Ephemeris Origin (CEO) in the Geocentric Celestial Reference System (GCRS) have been computed using the IAU 2000A precession-nutation ${ }^{\star}$. These expressions are for use in the new transformation between the GCRS and the International Terrestrial Reference System (ITRS) which is recommended by IAU Resolution B1.8. Various comparisons and numerical checks have been performed between the classical and the new transformations based on the IAU 2000A precession-nutation. These comparisons revealed necessary improvements to be applied to the classical form of the transformation in order to achieve the required level of accuracy. Once these improvements are applied, the consistency between the positions of the CIP in the GCRS corresponding to the classical and the new transformations is at a level of a few microarcseconds after one century. This work has demonstrated that the new method, in addition to providing an explicit separation between precession-nutation of the equator from Earth rotation, is more simple, compact and direct than the classical one, achieving accuracies at the level of a few microarcseconds with greatly reduced scope for accidental misuse. The resulting expressions for $X, Y$ and $s$ have been included in the IERS Conventions 2000. References for the numerical expressions are provided in Appendix C.
\end{abstract}

Key words. astrometry - reference systems - ephemerides - time

\section{Introduction}

This paper presents expressions for the position of the Celestial Intermediate Pole (CIP) and the Celestial Ephemeris Origin (CEO) in the Geocentric Celestial Reference System (GCRS) which have been computed using the IAU 2000A precession-nutation and the theoretical formulations developed in Capitaine et al. (2000).

The position of the CIP in the GCRS is provided by the coordinates $X$ and $Y$, which are the $x$ and $y$ components of the CIP unit vector. The coordinates are functions of the combined precession and nutation, together with the coupling effects between precession and nutation, and include also the frame rotation between the precession-nutation model at J2000 and the GCRS.

We will use the term "right ascension" in a more generic way than is strictly correct. In this paper, right ascension simply means the longitude coordinate of any Earth-pole-based

Send offprint requests to: N. Capitaine,

e-mail: capitaine@syrte.obspm.fr

* Tables for the series for $X, Y$ and $s+X Y / 2$ are available only in electronic form, at the CDS via anonymous ftp to cdsarc.u-strasbg.fr (130.79.128.5) or via

http://cdsweb.u-strasbg.fr/cgi-bin/qcat? J/A+A/400/1145 celestial coordinate system, and so may be referred to the $\mathrm{CEO}$ as well as some sort of equinox. The CEO is the origin of right ascension in the new system. Its counterpart in the classical system, namely the equinox, is defined geometrically. The two defining planes, the equator and ecliptic, are both in motion and not at right-angles, and consequently the motion of the equinox across the celestial sphere is complex (and comparatively rapid). The CEO, which is the non-rotating origin (Guinot 1979), in contrast is defined kinematically: from one moment to the next, the CEO moves only at right-angles to the instantaneous equator, and the ecliptic is not involved. This almost complete separation between the treatment of the precessing-nutating pole and the origin of right ascension leads to a much simpler relationship between stellar hour angles and Universal Time.

The concept of the CEO can be considered as extending that of a "uniform equinox" (Atkinson \& Sadler 1951) by eliminating not only the equation of the equinoxes in Sidereal time, as did the uniform equinox, but its precessional counterpart in the Greenwich mean sidereal time (GMST) formula itself. This nearly eliminates the dependence of the origin of right ascension, which is just defined by a convention, from the precession-nutation motion. The kinematical definition of the CEO can also be considered as extending the concept of 
“departure point" (Newcomb 1895) currently used in celestial mechanics or satellite geodesy.

The CEO's evolution with time depends on $s$, the difference between the GCRS and instantaneous right ascensions of the ascending node of the instantaneous equator. The quantity $s$ is defined by an integral that involves the path of the CIP, but the evolution of the CEO is both slow and insensitive to details of the CIP's motion. In the century 2000-2100, the CEO moves only 66 mas in GCRS right ascension.

The purpose of this paper is to provide positions of the CIP and the CEO computed to an accuracy of a few microarcseconds over a time span of a few hundred years, meeting the requirements of high-accuracy applications. The use of the $X, Y$ and $s$ parameters for long-term studies would require a different development in trigonometric functions of the precession angles, which will not be discussed here.

Developments of $X, Y$ and $s$ at microarcsecond accuracy, valid over an interval of several centuries, can be provided as polynomials in $t$ plus a series of Poisson terms. This avoids complicated multi-stage classical transformations for $X$ and $Y$ and a numerical integration for $s$.

The precession and nutation quantities used in these computations are the MHB 2000 nutation series and the IAU 1976 precession with corrected rates in longitude and obliquity (Mathews et al. 2002), together with the associated values for the CIP offsets at J2000 with respect to the GCRS pole. This use of the IAU $2000 \mathrm{~A}$ model is in agreement with the recommendation of IAU Resolution B1.6, and is supported through the IERS Conventions. The present implementation of the expressions is thus based on a semi-analytical development of nutation, which requires the use of trigonometric series, valid over a limited interval of time. Moreover, whereas more sophisticated developments of the precession quantities are available (Simon et al. 1994; Williams 1994), the present study makes use of the IAU 2000 precession, the rates of which are estimated from the most recent VLBI data.

The quantity used for the offset in right ascension results from simultaneous analysis of VLBI and LLR data (Chapront et al. 2002). The Fourier and Poisson series were handled using the GREGOIRE software package, capable of rapid manipulation of large series.

The formulations are given in Sect. 2, and the models and software upon which the calculations are based are described in Sect. 3. Expressions for the CIP coordinates $X$ and $Y$ are introduced in Sect. 4, along with internal comparisons between the series developments and classical formulations. Section 5 gives the expression for the quantity $s$ that positions the CEO on the equator, plus comparisons with direct numerical integrations. Finally, Sect. 6 presents external comparisons between the new and classical transformations, as well as a comparison with "observed" $X$ and $Y$. References for the numerical expressions are provided in Appendix C.

\section{The new paradigm versus classical paradigm}

Equivalent transformations from the GCRS to the frame of date can be provided using either the classical (equinox-based) paradigm or the new (CEO-based) paradigm. The pole for both methods is the CIP.

The conventional classical paradigm for this transformation uses a sequence of six consecutive rotations, for precession and nutation, followed by a further one for Earth rotation. The transformation is based on the developments as function of time of the precession angles $\zeta_{\mathrm{A}}, \theta_{\mathrm{A}}, z_{\mathrm{A}}$ (Lieske et al. 1977) and of the nutation angles $\Delta \psi$ in longitude and $\Delta \epsilon$ in obliquity referred to the ecliptic of date. It uses the relationship between Greenwich sidereal time and UT1, obtained from the GMST at date $t$ and the equation of the equinoxes. The frame bias has up to now been neglected as a rule, but is sometimes included as part of the nutation quantities.

The new paradigm (16), in contrast, keeps the concepts of the pole position and Earth rotation separate, leading to a cleaner implementation that has particular advantages for highaccuracy Earth rotation studies. The CIP position $X, Y$ is used directly, taking rigorous account of frame bias and dealing with precession-nutation. A third number, the small and slowlyvarying quantity $s$, defines an origin of right ascension that matches an Earth rotation angle the time derivative of which is equal to the Earth's angular velocity. The complicated formulas for GMST and the equation of the equinoxes are eliminated.

As already pointed out, the classical and new paradigms share a common pole, the CIP, specified in terms of the $X$ and $Y$ components of its unit vector in the GCRS. In the classical paradigm (see (14)), these components are readily obtained simply by forming the frame bias and precession-nutation matrix and extracting elements $(3,1)$ and $(3,2)$.

\section{Formulations}

The position of the CIP in the GCRS, as defined by IAU Resolution B1.7, is a function of frame bias, precession and nutation. The CIP coordinates can be obtained by evaluating the corresponding sequence of nine or ten coordinate rotations, but a convenient alternative is to use the simple expressions for $X$ and $Y$ given in Capitaine (1990) and Capitaine et al. (2000). The following expressions for $X$ and $Y$ are accurate to better than 1 microarcsecond at current epochs:

$X=\bar{X}+\xi_{0}-d \alpha_{0} \bar{Y}$,

$Y=\bar{Y}+\eta_{0}+d \alpha_{0} \bar{X}$

where $\xi_{0}$ and $\eta_{0}$ are the celestial pole offsets at epoch and $d \alpha_{0}$ the right ascension of the mean equinox at epoch in the GCRS. Quantities $\bar{X}$ and $\bar{Y}$ are given by:

$\bar{X}=\sin \omega \sin \psi$,

$\bar{Y}=-\sin \epsilon_{0} \cos \omega+\cos \epsilon_{0} \sin \omega \cos \psi$,

where $\epsilon_{0}$ is the obliquity of the ecliptic at J2000, $\omega$ is the inclination of the true equator of date on the fixed ecliptic of epoch and $\psi$ is the longitude, on the ecliptic of epoch, of the node of the true equator of date on the fixed ecliptic of epoch; these quantities are such that:

$\omega=\omega_{\mathrm{A}}+\Delta \epsilon_{1}, \quad \psi=\psi_{\mathrm{A}}+\Delta \psi_{1}$, 
where $\psi_{\mathrm{A}}$ and $\omega_{\mathrm{A}}$ are the precession quantities in longitude and obliquity (Lieske et al. 1977) referred to the ecliptic of epoch and $\Delta \psi_{1}, \Delta \epsilon_{1}$ are the nutation angles in longitude and obliquity referred to the ecliptic of epoch. $\Delta \psi_{1}, \Delta \epsilon_{1}$ can be obtained from the nutation angles $\Delta \psi, \Delta \epsilon$ in longitude and obliquity referred to the ecliptic of date, using the following formula from Aoki \& Kinoshita (1983), which has been verified to provide an accuracy better than one microarcsecond for a century:

$$
\begin{aligned}
\Delta \psi_{1} & =\frac{\left(\Delta \psi \sin \epsilon_{\mathrm{A}} \cos \chi_{\mathrm{A}}-\Delta \epsilon \sin \chi_{\mathrm{A}}\right)}{\sin \omega_{\mathrm{A}}}, \\
\Delta \epsilon_{1} & =\Delta \psi \sin \epsilon_{\mathrm{A}} \sin \chi_{\mathrm{A}}+\Delta \epsilon \cos \chi_{\mathrm{A}},
\end{aligned}
$$

$\epsilon_{\mathrm{A}}$ being the precession quantity in obliquity referred to ecliptic of date and $\chi_{\mathrm{A}}$ the precession quantity for planetary precession along the equator (Lieske et al. 1977).

In the following the numerical expressions of the direction cosines $X$ and $Y$ are multiplied by the factor $1296000^{\prime \prime} / 2 \pi$, in order to provide in arcseconds the value of the corresponding "angles" with respect to the polar axis of GCRS. These quantities can be developed at microarcsecond accuracy as polynomials in $t$ plus a series of Poisson terms, valid over an interval of several centuries: see expressions (11) and (12).

The parameter $t$, used in the following expressions, is defined by:

$t=(T T-2000$ January $1 \mathrm{~d} 12 \mathrm{~h} T T) / 36525$.

with $T T$ in days.

The numerical values of the coefficients of the polynomial part of $X$ and $Y$ are derived from the development as a function of time of the precession in longitude and obliquity and pole offset at $\mathbf{J} 2000.0$ and the amplitudes $\left(a_{s, j}\right)_{i},\left(a_{c, j}\right)_{i},\left(b_{s, j}\right)_{i}$, $\left(b_{c, j}\right)_{i}$, for $j=0,1,2 \ldots$ are derived from the amplitudes of the precession and nutation series. The amplitudes $\left(a_{s, 0}\right)_{i},\left(b_{c, 0}\right)_{i}$ are equal to the amplitudes of the in-phase terms of nutation in longitude $\left(\times \sin \epsilon_{0}\right)$ and obliquity respectively except for a few terms which contain a contribution from crossed-nutation effects.

In a finite displacement of the CIP between dates $t_{0}$ and $t$, the motion of the CEO is provided by the quantity $s(t)$ defined by Capitaine et al. (1986). It is the composite arc, which expresses the difference between the arc on the equator of date, from the CEO to $N$, and the arc, on the equator of the GCRS, from the origin, $O$, of right ascensions in the GCRS to $N, N$ being the node of the equator of date on the equator of the GCRS.

To the same accuracy as for the previous quantities, the quantity $s$ positioning the $\mathrm{CEO}$ on the equator is given by:

$s(t)=-\frac{1}{2}\left[X(t) Y(t)-X\left(t_{0}\right) Y\left(t_{0}\right)\right]+\int_{t_{0}}^{t} \dot{X}(t) Y(t) \mathrm{d} t$,

using, by convention, $\sigma_{0} N_{0}=\Sigma_{0} N_{0}$, where $\sigma_{0}$ and $\Sigma_{0}$ are the positions of the CEO at J2000 and the $x$-origin of the GCRS respectively and $N_{0}$ is the ascending node of the equator at $\mathrm{J} 2000$ in the equator of the GCRS.

\section{Models and calculation procedure}

The calculation procedure is based on the IAU 2000A precession-nutation and pole offset models and the use of the GREGOIRE software package.

\subsection{The IAU 2000A precession-nutation model}

The IAU 2000A Precession-Nutation model has been adopted by the IAU (Resolution B1.6) to replace the IAU 1976 Precession (Lieske et al. 1977) and the IAU 1980 Theory of Nutation (Wahr 1981; Seidelmann 1982). See Dehant et al. (1999) for more detail. This model (MHB2000) has been developed by Mathews et al. (2002) by direct solution of the linearized dynamical equation of the wobble-nutation problem of the Earth and makes use of estimated values of seven of the parameters appearing in the theory. The nonrigid model improves the IAU 1980 Theory of nutation by taking into account the effect of mantle anelasticity, ocean tides, electromagnetic couplings produced between the fluid outer core and the mantle as well as between the solid inner core and fluid outer core and the consideration of non-linear terms which have hitherto been ignored in this type of formulation. The basic Earth parameters have been estimated from a least squares fit to VLBI data set of "celestial pole offsets" obtained from VLBI measurements (Herring et al. 2002). The nutation series has been generated by the convolution of the MHB2000 transfer function with the Rigid-Earth nutation series REN-2000 (Souchay et al. 1999), rescaled to account for the change in the dynamical ellipticity of the Earth implied by the observed correction to the lunisolar precession of the equator.

The resulting nutation series includes 678 lunisolar terms and 687 planetary terms and provides the direction of the celestial pole in the GCRS with an accuracy of 0.2 mas. The series includes the geodesic nutation (Fukushima 1991). On the other hand, the nutational-type motions due to excitation of free modes, such as the Free Core Nutation (FCN), which cannot be predicted rigorously, are not included in the IAU 2000A model.

The IAU 2000 nutation series is associated with improved numerical values for the precession rate of the equator in longitude and obliquity:

$$
\begin{aligned}
& \delta \psi_{\mathrm{A}}=(-0.29965 \pm 0.00040)^{\prime \prime} / \mathrm{c} \\
& \delta \omega_{\mathrm{A}}=(-0.02524 \pm 0.00010)^{\prime \prime} / \mathrm{c}
\end{aligned}
$$

The IAU 2000A Fortran code (IERS Conventions 2000, Chap. 5) returns the precession corrections themselves as well as combined with the nutations, giving the user choice in how they are to be employed. The most straightforward approach is simply to add them to the Lieske et al. (1977) expressions for $\psi_{\mathrm{A}}$ and $\epsilon_{\mathrm{A}}$. These corrected angles can then be used in the formulation given in Sect. 2. Applications requiring the classical precession matrix can use the corrected $\psi_{\mathrm{A}}$ and $\epsilon_{\mathrm{A}}$ in the four-angle formulation: $R_{3}\left(\chi_{\mathrm{A}}\right) R_{1}\left(-\omega_{\mathrm{A}}\right) R_{3}\left(-\psi_{\mathrm{A}}\right) R_{1}\left(\epsilon_{0}\right)$.

\subsection{Offsets at J2000}

The IAU 2000 nutation series is associated with the constant offsets $\delta \psi_{0}$ and $\delta \epsilon_{0}$ of the direction of the CIP at J2000.0 from the direction of the pole of the GCRS,

$\delta \psi_{0}=(-0.0417750 \pm 0.000025)^{\prime \prime}$
$\delta \epsilon_{0}=(-0.0068192 \pm 0.0000100)^{\prime \prime}$ 


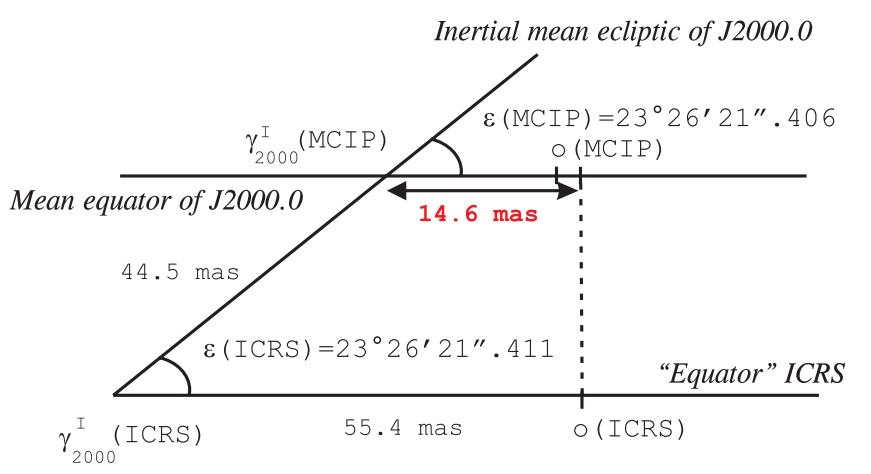

Fig. 1. Offset in right ascension (Chapront et al. 2002).

from which the following values, $\xi_{0}$ and $\eta_{0}$, for the celestial pole offsets at epoch, have been adopted:

$\xi_{0}=(-0.016617 \pm 0.000010)^{\prime \prime}$

$\eta_{0}=(-0.006819 \pm 0.000010)^{\prime \prime}$.

The quantity $\mathrm{d} \alpha_{0}$, provided by (10), used for the offset in right ascension was obtained from simultaneous analysis of VLBI and LLR data (Chapront et al. 2002). The sign convention is such that 14.6 mas is minus the GCRS right ascension of the mean equinox at J2000 (see Fig. 1). The mean equinox of epoch to be considered is not the "rotational dynamical mean equinox of J2000.0" (Standish 1981) corresponding to a computation with respect to a rotating ecliptic, as used in the past, but the inertial dynamical mean equinox of J2000.0, corresponding to a computation with respect to a fixed ecliptic to which the recent numerical or analytical solutions refer.

$d \alpha_{0}=(-0.0146 \pm 0.0005)^{\prime \prime}$.

Note that this rotation does not cause 14.6 mas shifts in the right ascensions of stars in either the GCRS or the intermediate frame. It merely re-orients the underlying classical precessionnutation model, eliminating a small but spurious distortion that would otherwise be present. The 14.6 mas rotation is canceled by compensating changes to the new sidereal time formulas (Capitaine et al. 2003).

\subsection{The software GREGOIRE}

The precession quantities are time polynomials; the nutation quantities are Poisson series, i.e. Fourier series whose coefficients are time expansions. Our main goal is to build explicit expressions for $X, Y$ and $s$ which can be used instead of precession and nutation angles. The manipulation of these quantities as they appear in formulas (1) to (4) in a closed form requires an algebraic tool that handles Poisson series and provides addition, multiplication, differentiation, integration and other operations such as Taylor's developments, inverse of a series, etc. The Poisson series depend on literal arguments (lunar angles, solar and planetary longitudes and also precession). The coefficients are time polynomials in $t^{k}$ truncated to a maximum power $k$, with the condition that those smaller than $1 \mu$ as after 2 centuries are to be neglected. The tool which has been used is GREGOIRE, a software package devoted to Poisson series manipulations. It allows a large numbers of Fourier arguments $(\leq 32)$ and a reasonable maximum time power $(\leq 10)$, depending on the largest dimension of the series. It contains a wide number of facilities to perform all the usual operations via simple scripts. In particular it allows the computation of an ephemeris for given values of the time which is explicit in the coefficients and implicit in the arguments. This tool is essential to check the final accuracy of the results. The software is described in more detail in Appendix A.

\section{Position of the CIP in the GCRS}

\subsection{Expressions for the coordinates $X$ and $Y$}

The expressions of $X$ and $Y$ are derived from (1) to (4) based on the IAU 2000 precession and nutation. The numerical values of $\left(a_{s, j}\right)_{i},\left(a_{c, j}\right)_{i},\left(b_{c, j}\right)_{i},\left(b_{s, j}\right)_{i}$ for $j=0,1,2$ are available as tables in electronic form (see Appendix C), of which the largest terms are listed in Table 1. For reason of continuity with the IAU 1980 nutation theory, the in-phase terms are sine terms in longitude $\left(a_{\mathrm{s}, 0}\right)_{i}$ and cosine terms in obliquity $\left(b_{\mathrm{c}, 0}\right)_{i}$. These expressions have the following form:

$$
\begin{aligned}
X= & -0 .^{\prime} 016617+2004^{\prime \prime} 191743 t-0^{\prime} \cdot 4272190 t^{2} \\
& -0{ }^{\prime} 1986205 t^{3}-00^{\prime} 0000460 t^{4}+00^{\prime} 0000060 t^{5} \\
& +\sum_{i}\left[\left(a_{\mathrm{s}, 0}\right)_{i} \sin (\mathrm{ARG})+\left(a_{\mathrm{c}, 0}\right)_{i} \cos (\mathrm{ARG})\right] \\
& +\sum_{i}\left[\left(a_{\mathrm{s}, 1}\right)_{i} t \sin (\mathrm{ARG})+\left(a_{\mathrm{c}, 1}\right)_{i} t \cos (\mathrm{ARG})\right] \\
& +\sum_{i}\left[\left(a_{\mathrm{s}, 2}\right)_{i} t^{2} \sin (\mathrm{ARG})+\left(a_{\mathrm{c}, 2}\right)_{i} t^{2} \cos (\mathrm{ARG})\right]+\cdots \\
Y= & -0 .^{\prime} 006951-0{ }^{\prime} 025382 t-22^{\prime \prime} 4072510 t^{2} \\
& +0 .^{\prime} 0018423 t^{3}+0 .^{\prime} 0011131 t^{4}+0{ }^{\prime} 00000099 t^{5} \\
& +\sum_{i}\left[\left(b_{\mathrm{c}, 0}\right)_{i} \cos (\mathrm{ARG})+\left(b_{\mathrm{s}, 0}\right)_{i} \sin (\mathrm{ARG})\right] \\
& +\sum_{i}\left[\left(b_{\mathrm{c}, 1}\right)_{i} t \cos (\mathrm{ARG})+\left(b_{\mathrm{s}, 1}\right)_{i} t \sin (\mathrm{ARG})\right] \\
& +\sum_{i}\left[\left(b_{\mathrm{c}, 2}\right)_{i} t^{2} \cos (\mathrm{ARG})+\left(b_{\mathrm{s}, 2}\right)_{i} t^{2} \sin (\mathrm{ARG})\right]+\cdots
\end{aligned}
$$

ARG stands for various combinations of the fundamental arguments of the nutation theory, including both luni-solar and planetary terms (see Appendix B).

\subsection{Internal comparisons}

In order to check the developments as function of time of the quantities $X$ and $Y$, an internal comparison in the time domain over 4 centuries around $\mathbf{J} 2000$ has been performed (see Fig. 2) between ephemerides using the developments as functions of time for $X$ and $Y$ (index $a$ ) and the values at the same dates based on the analytical formulation as functions of the classical precession and nutation quantities (index $b$ ).

The differences are less than five microarcseconds over the considered interval.

Spectra of the differences show no amplitude greater than $0.3 \mu$ as (see Fig. 3). 
Table 1. Largest non-polynomial terms in the development of $X(t)$ (formula (11)) (top part) and $Y(t)$ (formula (12)) (bottom part) compatible with IAU 2000A precession-nutation model (unit $\mu$ as). The expressions for the fundamental arguments appearing in columns 4 to 17 are provided in Appendix B.

\begin{tabular}{|c|c|c|c|c|c|c|c|c|c|c|c|c|c|c|c|c|}
\hline$i$ & $\left(a_{s, 0}\right)_{i}$ & $\left(a_{c, 0}\right)_{i}$ & $l$ & $l^{\prime}$ & $F$ & $D$ & $\Omega$ & $L_{\mathrm{Me}}$ & $L_{\mathrm{Ve}}$ & $L_{\mathrm{E}}$ & $L_{\mathrm{Ma}}$ & $L_{\mathrm{J}}$ & $L_{\mathrm{Sa}}$ & $L_{\mathrm{U}}$ & $L_{\mathrm{Ne}}$ & $p_{\mathrm{A}}$ \\
\hline 1 & -6844318.44 & 1328.67 & 0 & 0 & 0 & 0 & 1 & 0 & 0 & 0 & 0 & 0 & 0 & 0 & 0 & 0 \\
\hline 2 & -523908.04 & -544.76 & 0 & 0 & 2 & -2 & 2 & 0 & 0 & 0 & 0 & 0 & 0 & 0 & 0 & 0 \\
\hline 3 & -90552.22 & 111.23 & 0 & 0 & 2 & 0 & 2 & 0 & 0 & 0 & 0 & 0 & 0 & 0 & 0 & 0 \\
\hline 4 & 82168.76 & -27.64 & 0 & 0 & 0 & 0 & 2 & 0 & 0 & 0 & 0 & 0 & 0 & 0 & 0 & 0 \\
\hline 5 & 58707.02 & 470.05 & 0 & 1 & 0 & 0 & 0 & 0 & 0 & 0 & 0 & 0 & 0 & 0 & 0 & 0 \\
\hline \multicolumn{17}{|l|}{..... } \\
\hline$i$ & $\left(a_{s, 1}\right)_{i}$ & $\left(a_{c, 1}\right)_{i}$ & $l$ & $l^{\prime}$ & $F$ & $D$ & $\Omega$ & $L_{\mathrm{Me}}$ & $L_{\mathrm{Ve}}$ & $L_{\mathrm{E}}$ & $L_{\mathrm{Ma}}$ & $L_{\mathrm{J}}$ & $L_{\mathrm{Sa}}$ & $L_{\mathrm{U}}$ & $L_{\mathrm{Ne}}$ & $p_{\mathrm{A}}$ \\
\hline 1307 & -3328.48 & 205833.15 & 0 & 0 & 0 & 0 & 1 & 0 & 0 & 0 & 0 & 0 & 0 & 0 & 0 & 0 \\
\hline 1308 & 197.53 & 12814.01 & 0 & 0 & 2 & -2 & 2 & 0 & 0 & 0 & 0 & 0 & 0 & 0 & 0 & 0 \\
\hline 1309 & 41.19 & 2187.91 & 0 & 0 & 2 & 0 & 2 & 0 & 0 & 0 & 0 & 0 & 0 & 0 & 0 & 0 \\
\hline \multicolumn{17}{|l|}{..... } \\
\hline$i$ & $\left(b_{s, 0}\right)_{i}$ & $\left(b_{c, 0}\right)_{i}$ & $l$ & $l^{\prime}$ & $F$ & $D$ & $\Omega$ & $L_{M e}$ & $L_{\mathrm{Ve}}$ & $L_{\mathrm{E}}$ & $L_{\mathrm{Ma}}$ & $L_{\mathrm{J}}$ & $L_{\mathrm{Sa}}$ & $L_{\mathrm{U}}$ & $L_{\mathrm{Ne}}$ & $p_{\mathrm{A}}$ \\
\hline 1 & 1538.18 & 9205236.26 & 0 & 0 & 0 & 0 & 1 & 0 & 0 & 0 & 0 & 0 & 0 & 0 & 0 & 0 \\
\hline 2 & -458.66 & 573033.42 & 0 & 0 & 2 & -2 & 2 & 0 & 0 & 0 & 0 & 0 & 0 & 0 & 0 & 0 \\
\hline 3 & 137.41 & 97846.69 & 0 & 0 & 2 & 0 & 2 & 0 & 0 & 0 & 0 & 0 & 0 & 0 & 0 & 0 \\
\hline 4 & -29.05 & -89618.24 & 0 & 0 & 0 & 0 & 2 & 0 & 0 & 0 & 0 & 0 & 0 & 0 & 0 & 0 \\
\hline 5 & -17.40 & 22438.42 & 0 & 1 & 2 & -2 & 2 & 0 & 0 & 0 & 0 & 0 & 0 & 0 & 0 & 0 \\
\hline \multicolumn{17}{|l|}{..... } \\
\hline$i$ & $\left(b_{s, 1}\right)_{i}$ & $\left(b_{c, 1}\right)_{i}$ & $l$ & $l^{\prime}$ & $F$ & $D$ & $\Omega$ & $L_{\mathrm{Me}}$ & $L_{\mathrm{Ve}}$ & $L_{E}$ & $L_{\mathrm{Ma}}$ & $L_{\mathrm{J}}$ & $L_{\mathrm{Sa}}$ & $L_{\mathrm{U}}$ & $L_{\mathrm{Ne}}$ & $p_{\mathrm{A}}$ \\
\hline 963 & 153041.82 & 878.89 & 0 & 0 & 0 & 0 & 1 & 0 & 0 & 0 & 0 & 0 & 0 & 0 & 0 & 0 \\
\hline 964 & 11714.49 & -289.32 & 0 & 0 & 2 & -2 & 2 & 0 & 0 & 0 & 0 & 0 & 0 & 0 & 0 & 0 \\
\hline 965 & 2024.68 & -50.99 & 0 & 0 & 2 & 0 & 2 & 0 & 0 & 0 & 0 & 0 & 0 & 0 & 0 & 0 \\
\hline$\ldots$ & & & & & & & & & & & & & & & & \\
\hline
\end{tabular}

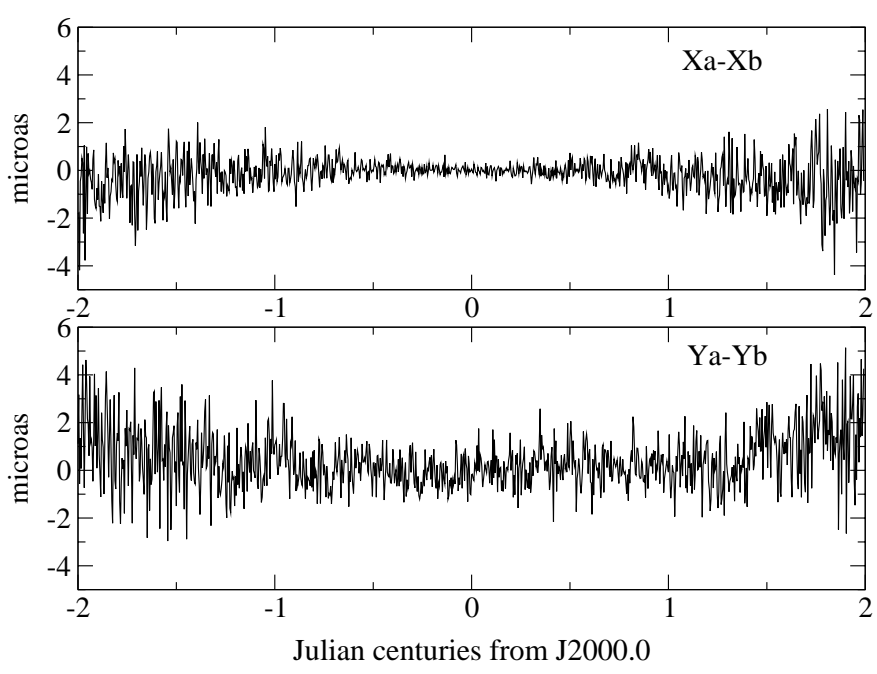

Fig. 2. Comparison between the developments (11) and (12) of the quantities $X$ and $Y$ and ephemerides for the analytical formulation as function of the combined IAU 2000A precession-nutation quantities.

Plots of the differences (see Fig. 4) over a longer interval (4 centuries around J2000) demonstrate the interval of validity of the developments ( 2 centuries).

\section{Position of the CEO in the GCRS}

\subsection{Expression for the quantity $s$}

The quantity $s$ is used along with $X$ and $Y$ to predict the position of the CEO as a function of time. By convention, $s$ is the

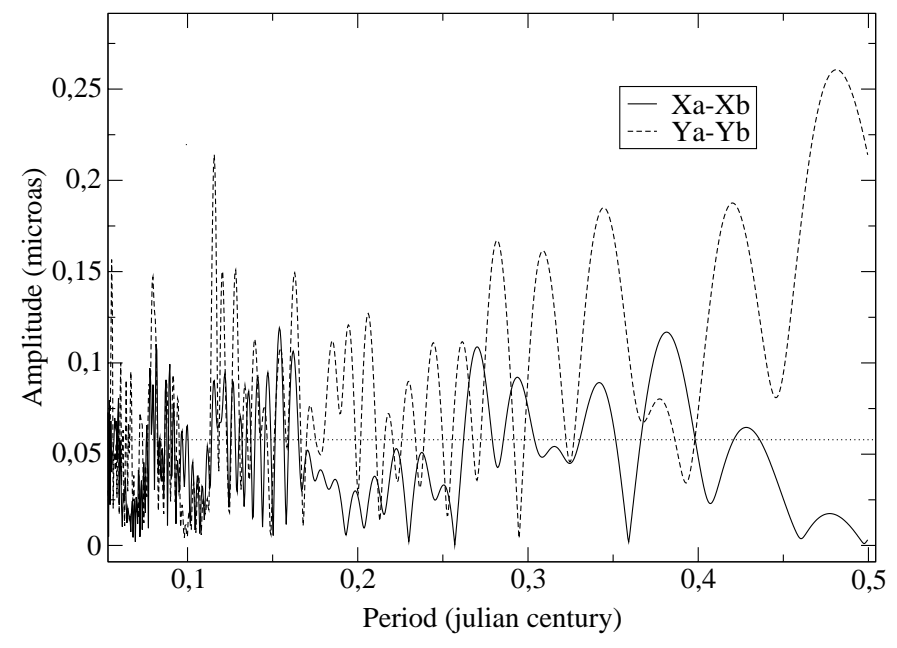

Fig. 3. Spectrum of the differences (displayed in Fig. 2) in $X$ and $Y$ coordinates from 0 to 0.5 centuries.

difference, small at present, between the GCRS and instantaneous right ascensions of the ascending node of the CIP equator on the GCRS equator.

The CEO is defined kinematically, and its position as a function of time depends on an integral that involves the motion of the CIP. The formulation given earlier (expression (6)) gives an approximation to $s$ directly.

In most applications, a numerical integration procedure is too computationally expensive to be practical, and so a direct formula for $s$ as a function of time is required. The numerical development is provided for the quantity $s+X Y / 2$, which 


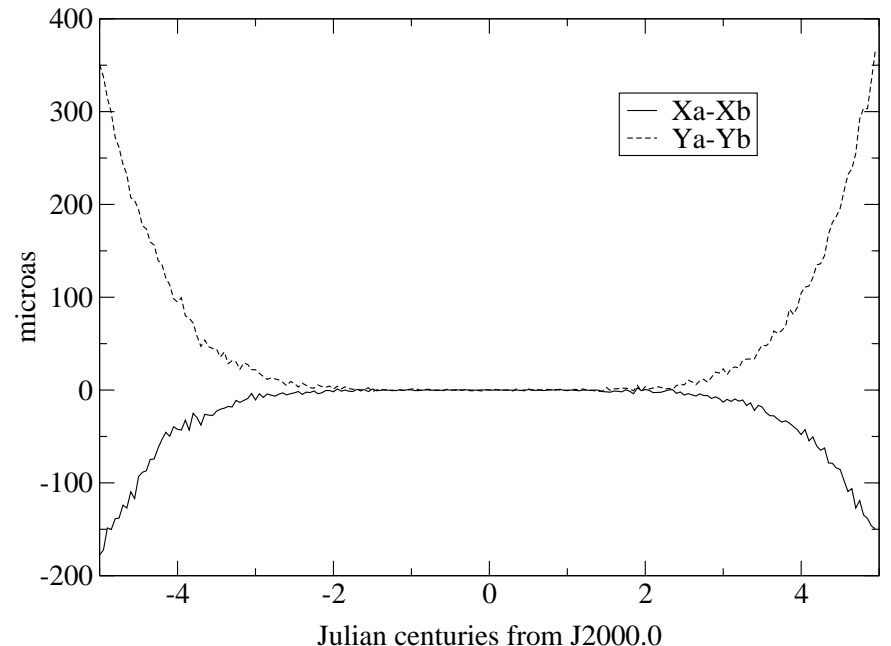

Fig. 4. Comparison between the development of the quantities $X$ and $Y$ over 4 centuries and ephemerides for the analytical formulation as functions of the combined IAU 2000A precession-nutation quantities.

requires fewer terms to reach the same accuracy than a direct development for $s$. Note that this quantity is equal, up to the $3 \mathrm{~d}$ order in $X$ and $Y$, to the GCRS right ascension of the CEO. The expression for $s+X Y / 2$ compatible with the IAU 2000A precession-nutation model, with all terms larger than $0.1 \mu$ as, is available in electronic form (see Appendix C). The largest terms, with amplitudes greater than $10 \mu$ as, are as follows (unit $\mu$ as):

$$
\begin{aligned}
s(t)= & -X Y / 2+94+3808 t-120 t^{2}-72574 t^{3} \\
& -2641 \sin \Omega-64 \sin 2 \Omega \\
& -12 \sin (2 F-2 D+3 \Omega)-11 \sin (2 F-2 D+\Omega) \\
& +744 t^{2} \sin \Omega+57 t^{2} \sin (2 F-2 D+2 \Omega) \\
& +10 t^{2} \sin (2 F+2 \Omega) \\
& +28 t^{4}+15 t^{5}-22 t^{3} \cos \Omega,
\end{aligned}
$$

where $F, D$ and $\Omega$ are Delaunay's nutation arguments (see Appendix B).

The contribution of terms with very long periods (greater than 500 years) have been replaced by a contribution to the secular term which has been evaluated to $16 \mu \mathrm{as} / \mathrm{c}$.

It should be noted that the zero-point and the secular term in the development of $s$ have been fitted taking into account the need for continuity in UT1 (Capitaine et al. 2003).

\subsection{Comparison with a numerical computation of the quantity $s$ from observed position of the CIP}

The observed celestial pole offsets as derived from VLBI observations can be used to compute "observed" values for the CIP coordinates $X$ and $Y$. Such"observed" values are obtained by adding the values computed from the current IAU model for precession and nutation (1976 Precession and 1980 Nutation) for the quantities $X$ and $Y$ to the celestial pole offsets in $\mathrm{d} \psi \times \sin \epsilon_{0}$ and $\mathrm{d} \epsilon$ for each date (at intervals of 1 day from 1980 to 2002) of the IERS CO4 series. The development of the quantity $s$ as a function of time has been compared with a

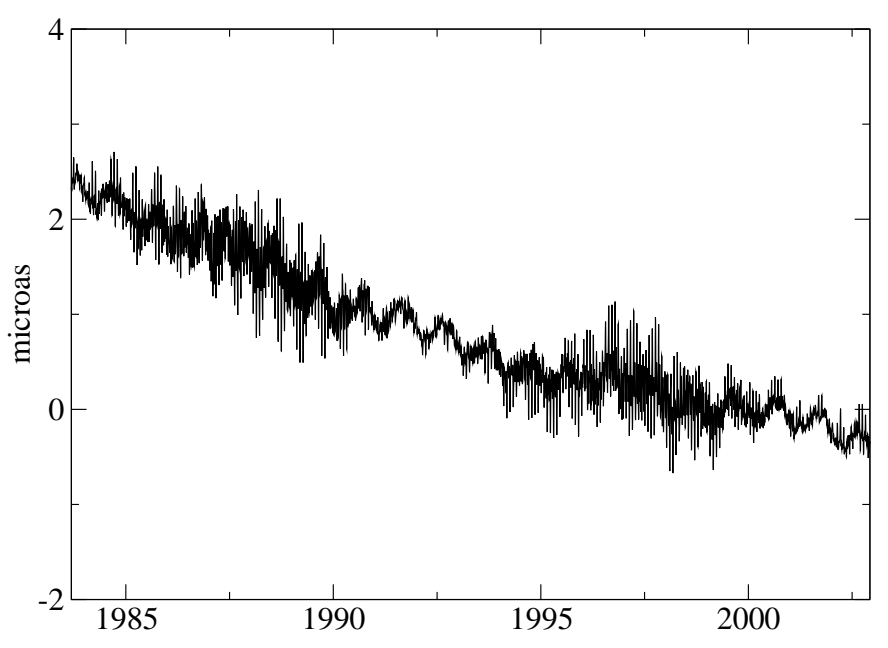

Fig. 5. Comparison between the development (13) of the quantity $s$ and numerical computation from IAU precession-nutation model and celestial pole offsets from IERS C04 series.

numerical quadrature of expression (6) based on this "observed" series for $X, Y$. Figure 5 provides the plot of differences during the duration of the series. The differences (which also reflect the deficiencies in the numerical computation based on an imperfect set of data) are less than $5 \mu$ as over the interval of the series. These differences can be reduced to $1 \mu$ as if the development of $s$ includes all the terms with amplitudes greater than $0.5 \mu$ as after one century, except for a trend of the order of $2 \mu$ as within the 20 -year interval.

\section{Numerical checks of the $X$ and $Y$ expressions}

\subsection{Comparison between new and classical expressions for $X$ and $Y$}

Comparing the new and classical transformations from GCRS to ITRS (omitting polar motion) can be done through the GCRS to ITRS rotation matrix, called $\mathbf{R}$ below. The classical method is to take the product of the individual rotation matrices for biases $(\mathbf{B})$, precession $(\mathbf{P})$, nutation $(\mathbf{N})$ and Earth rotation $\mathbf{T}$ :

$\mathbf{R}=\mathbf{T} \mathbf{N P B}$

where:

$\mathbf{B}=R_{1}\left(-\eta_{0}\right) \cdot R_{2}\left(\xi_{0}\right) \cdot R_{3}\left(d \alpha_{0}\right)$,

$\mathbf{P}=R_{3}\left(\chi_{\mathrm{A}}\right) \cdot R_{1}\left(-\omega_{\mathrm{A}}\right) \cdot R_{3}\left(-\psi_{\mathrm{A}}\right) \cdot R_{1}\left(\epsilon_{0}\right)$,

$\mathbf{N}=R_{1}\left(-\left[\epsilon_{\mathrm{A}}+\Delta \epsilon\right]\right) \cdot R_{3}(-\Delta \psi) \cdot R_{1}\left(\epsilon_{\mathrm{A}}\right)$

$\mathbf{T}=R_{3}(\mathrm{GST})$.

This classical transformation is equivalent to the (much more compact) new transformation:

$\mathbf{R}=R_{3}(\theta-E-s) \cdot R_{2}(d) \cdot R_{3}(E)$,

$\theta$ being the Earth Rotation Angle and $E$ and $d$ being derived from $X$ and $Y$ by:

$\begin{aligned} E & =\arctan (Y / X) \\ d & =\arctan \left(\left(\left(X^{2}+Y^{2}\right) /\left(1-X^{2}-Y^{2}\right)\right)^{1 / 2}\right) .\end{aligned}$ 


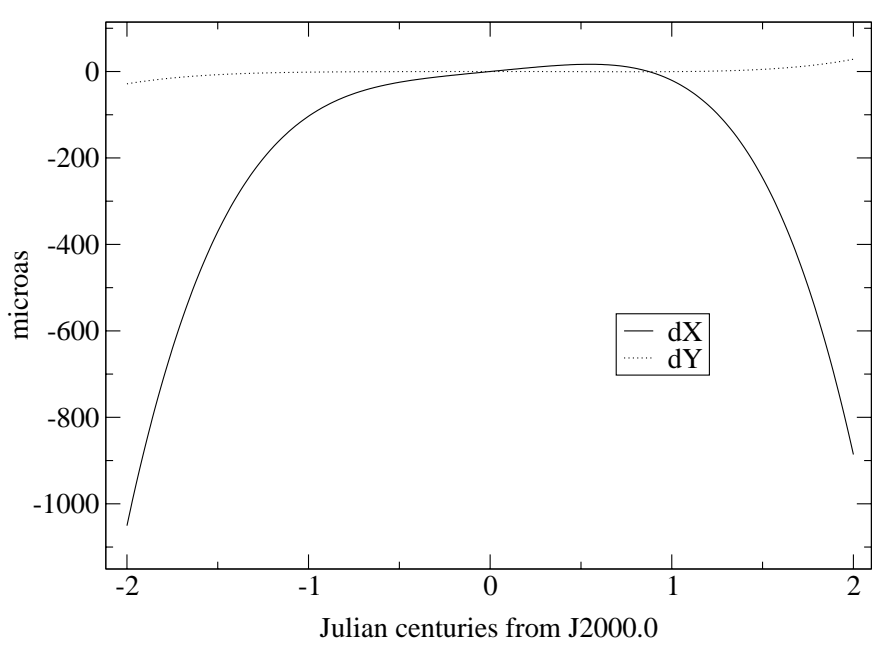

Fig. 6. Difference in the elements $(3,1)$ and $(3,2)$ of the classical $N P$ matrix between the most common two ways (using the 3-rotation or 4-rotation transformation) of forming the precession matrix.

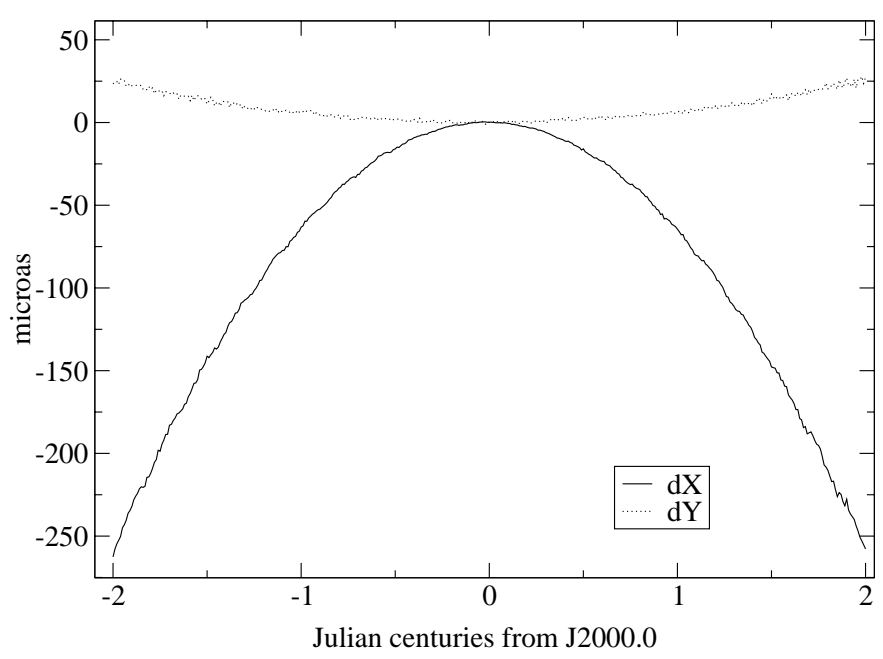

Fig. 7. Effect of using the IAU 2000A precession contribution rather than applying directly to $\psi_{\mathrm{A}}$ and $\omega_{\mathrm{A}}$.

For both the classical and new transformations, elements $(3,1)$ and $(3,2)$ of the $\mathbf{R}$ matrix are the CIP $X$ and $Y$.

Comparison of the series for $X, Y$ with these matrix elements revealed differences at one milliarcsecond level after two centuries. These differences have been explained by the different effects appearing in the classical transformation when the IAU 2000A nutation code is used (Wallace 2002), as described below:

- disagreement (at 1 mas level over 2 centuries) (see Fig. 6) between the two ways of forming the precession matrix using the angles of Lieske et al. (1977): the 3-rotation transformation based on the quantities $\zeta_{\mathrm{A}}, \theta_{\mathrm{A}}, z_{\mathrm{A}}$ and the 4-rotation one based on the quantities $\epsilon_{0}, \psi_{\mathrm{A}}, \omega_{\mathrm{A}}$ and $\chi_{\mathrm{A}}$,

- cross terms becoming significant after a few decades due to the use of the IAU 2000A "total" nutations that include the precession contributions, rather than applying the precession changes directly to $\psi_{\mathrm{A}}, \omega_{\mathrm{A}}$ and $\epsilon_{\mathrm{A}}$ (see Fig. 7),

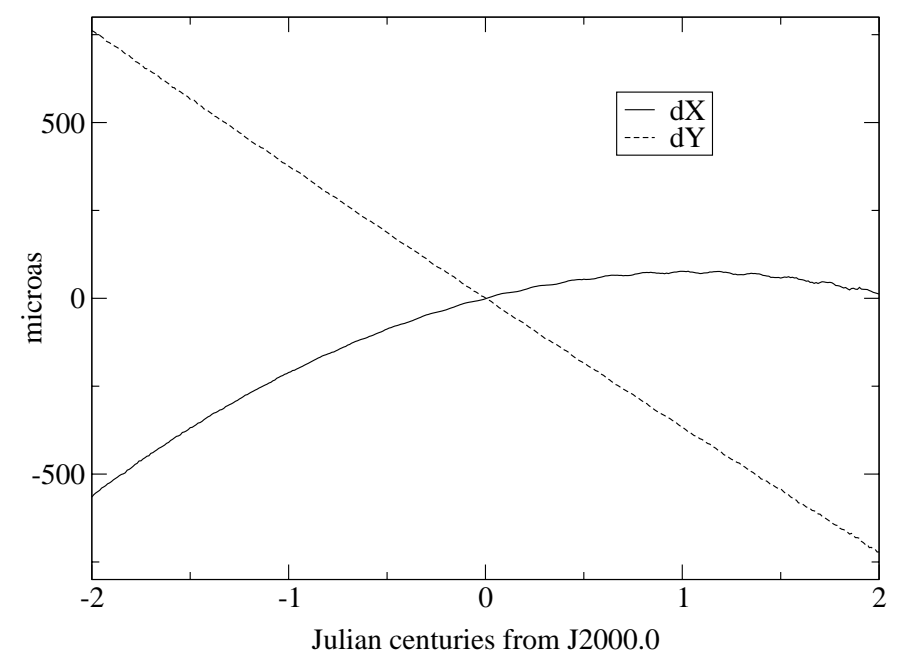

Fig. 8. Effect of using the IAU 2000 bias contributions rather than applying the ICRS to mean J2000 bias rigorously, as a separate rotation.

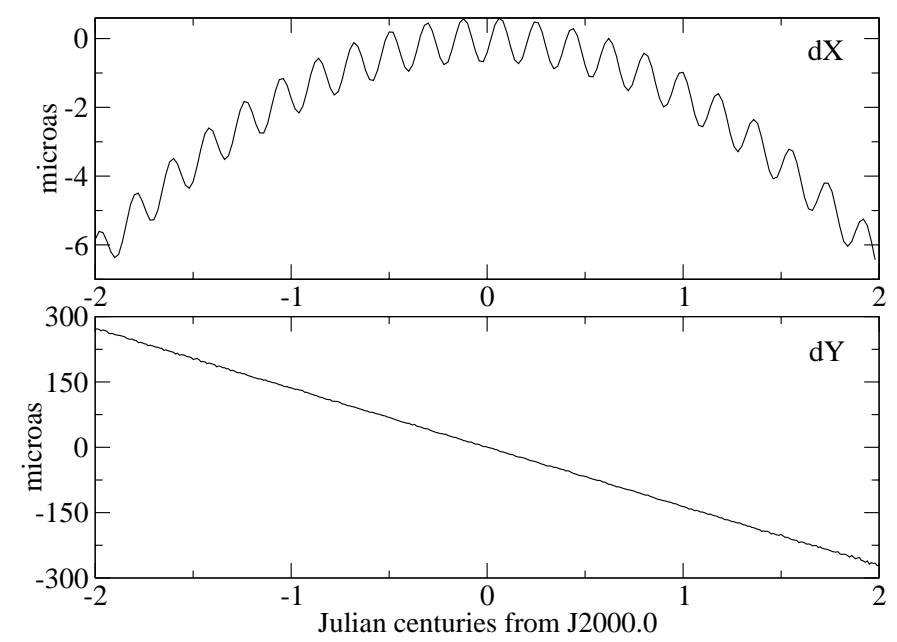

Fig. 9. Effect on $X$ and $Y$ of omitting the offset in right ascension at J2000.

- cross terms becoming significant after a few decades (see Fig. 8) due to the effect of using the IAU 2000A bias contributions rather than applying this offset at J2000 rigorously, as a separate rotation,

- cross-terms of non-negligible amplitudes over long periods of time appearing in $X, Y$, due to the effect of omitting the offset in right ascension at J2000, d $\alpha_{0}$ (see Fig. 9).

After correcting these effects, agreement to a few microarcseconds is achieved (see Fig. 10), but only because the "total" $\mathrm{d} \psi$ and $\mathrm{d} \epsilon$ from IAU 2000A have been rejected in favor of rigorous precession and bias corrections.

These comparisons have shown that although the classical method is capable of generating the precession-nutation, and hence the CIP $X, Y$ without inaccuracy, it is extremely easy when using it for high-accuracy applications to introduce subtle inconsistencies by accident. The pitfalls include performing the steps in the wrong order, omitting them, including them twice, introducing foreign quantities such as a "newer" obliquity, and so on. 


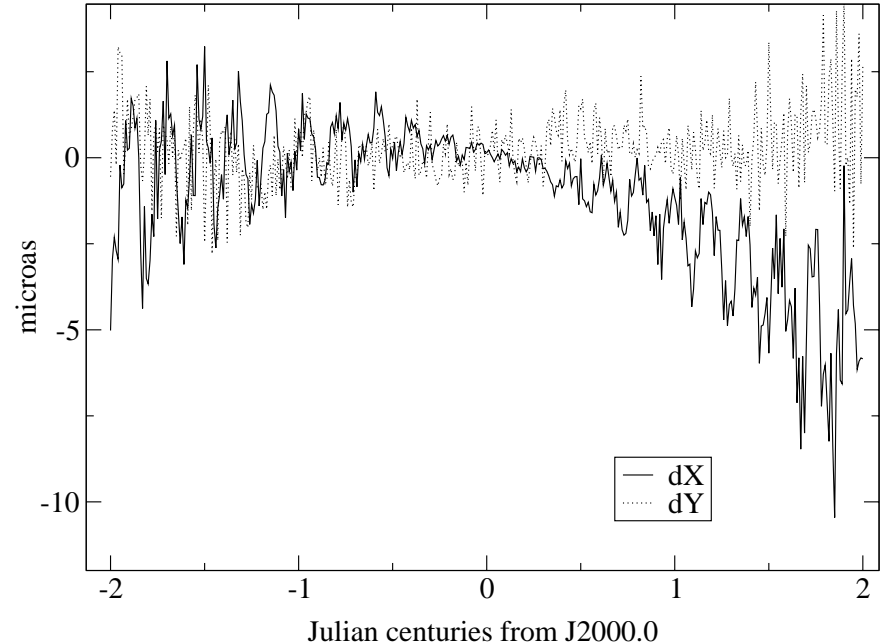

Fig. 10. Comparisons of the developments of $X$ and $Y$ with the series for $X, Y$ elements $(3,1)$ and $(3,2)$ of the classical $N P$ matrix.
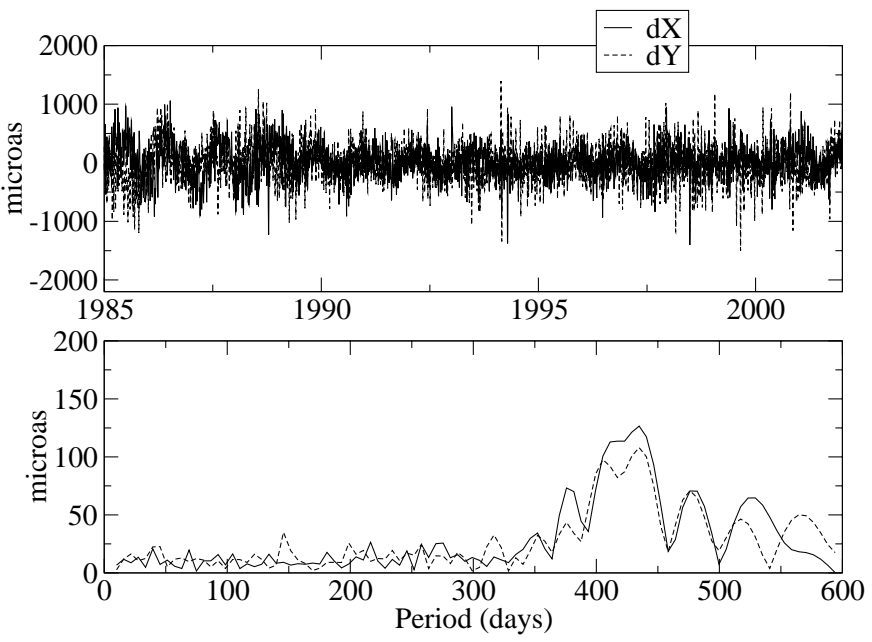

Fig. 11. Differences between "observed" $X, Y$ and the predicted values based on the IAU 2000A developments.

\subsection{Comparison of the expressions for $X$ and $Y$ with "observed" positions of the CIP}

As described in Sect. 6.2, "observed" values for $X$ and $Y$ can be provided by using the IAU 1976 precession, the IAU 1980 nutation and the IERS C04 celestial pole offsets as derived from VLBI observations. These "observed" positions of the CIP have been compared to the "computed" positions using the expressions for $X$ and $Y$ over the interval 1985-2002. The residuals plotted in Fig. 11 as well as the corresponding spectrum clearly reveal the presence of the time-varying FCN nutation modulation, the FCN mode being not included in the IAU 2000 precession-nutation (Herring et al. 2002), but being included in the IERS pole offsets.

\section{Conclusion}

Expressions have been provided for the position of the CIP and the CEO in the GCRS in agreement with the IAU 2000
Resolutions. The resulting expressions for $X, Y$ and $s$ have been included in the IERS Conventions 2000 (McCarthy \& Capitaine 2002). This work has demonstrated that the new method is more simple, compact and direct than the classical one, achieving accuracies at the level of a few microarcseconds with greatly reduced scope for misuse. The explicit separation between precession-nutation of the equator from Earth rotation is a further advantage of the new method.

\section{Appendix A: The software GREGOIRE}

GREGOIRE is a software package written in FORTRAN which handles Fourier and Poisson series which are represented by the general formula (A.1):

$$
\begin{array}{r}
S=\sum_{\left(i_{1}, i_{2}, \ldots, i_{n}\right)} \sum_{k=0}^{p} C_{i_{1}, i_{2}, \ldots, i_{n}}^{(k)} t^{k} \cos \left(i_{1} y_{1}+i_{2} y_{2}+\ldots+i_{n} y_{n}\right) \\
+S_{i_{1}, i_{2}, \ldots, i_{n}}^{(k)} t^{k} \sin \left(i_{1} y_{1}+i_{2} y_{2}+\ldots+i_{n} y_{n}\right)
\end{array}
$$

where $t$ is the time, which is explicit in case of Poisson series. The quantities $C_{i_{1}, i_{2}, \ldots, i_{n}}^{(k)}$ and $S_{i_{1}, i_{2}, \ldots, i_{n}}^{(k)}$ are numerical coefficients. The arguments of the series have formal components described by the set of integers: $\left(i_{1}, i_{2}, \ldots, i_{n}\right)$. The upper limits $p$ and $n$ are inputs of the program. GREGOIRE performs classical operations on series (addition, multiplication, input and output) which are limited by a precision $\epsilon$ such as (A.2):

$\left|C_{i_{1}, i_{2}, \ldots, i_{n}}^{(k)}\right|+\left|S_{i_{1}, i_{2}, \ldots, i_{n}}^{(k)}\right| \leq \epsilon$.

When the components of the arguments are linear functions of the time, differentiation, integration and time substitution are also available. The total number of numerical coefficients: $2 \times$ $p \times n$ can reach a maximum of about 100000 terms.

The algorithms to sort the series, to arrange the coefficients and to compress the data are very efficient to allow the rapid manipulation of large series of several thousand terms. Finally, the command interpreter helps the user to write scripts in an easy form. Constants and series are referred to by symbolic names. Other facilities, such as iteration, loops and indexes of series or constants are also available. Several functions are offered: to build an ephemeris, to compute a sine or a cosine of a series, to evaluate the inverse of a series, ... The user can also write his or her own routines, using the tools of GREGOIRE. The handling of series between memory and disk is transparent to the user. In the past, and in a more primitive form, this software has been extensively used at Bureau des Longitudes during the elaboration of planetary and lunar theories.

The precision in the manipulation of Poisson series is governed by two parameters:

1- the upper limit $p$ of the time power $t^{p}$ in the secular developments of the coefficients in (A.1)

$p$ is a truncation level of the secular developments. In the case of precession quantities like $\psi_{\mathrm{A}}$ or $\omega_{\mathrm{A}}$ this truncation level is 3 in Lieske's expressions. To maintain a precision of $1 \mu \mathrm{as}$ over two centuries after a time substitution, experience shows that one requires $p \leq 5$ in the computation of $X$ and $Y$.

2 - the limiting precision $\epsilon$ in the algebraic operations on the series (A.2) 
In Poisson series manipulations, one should be aware of the following difficulties in the product of two series $S=S_{1} \times S_{2}$ which is the most critical operation: a given argument of the product in $S$ is obtained by numerous contributions in $S_{1}$ and $S_{2}$ which accumulate. In order to ensure at the end of the product a precision $\epsilon$ in the resulting coefficient, because of the truncations, the intermediate operations have to be retained in an accumulator and tested to keep the internal numerical truncation smaller than $\epsilon$, say $\epsilon / 10$ for example. At the end of the product $S$ the coefficients in $S$ are limited to the condition (A.2), in order to avoid the proliferation of irrelevant terms (and eventually saturation of memory!). In our application we were guided by the final precision to be reached: $1 \mu$ as. In order to retain this precision after 2 centuries we carried out the computation to a precision of $0.01 \mu$ as with an internal limit even smaller. The bulkiness of the series is the practical limitation of the precision; this was not the case for the nutation series.

For the numerical substitution of time in the series and also for the integration and differentiation operations we need to specify numerical values for the frequencies (and also the phases) of the components of the arguments $y_{j}$ in (A.1). In the integration process some algebraic combinations of frequencies in the arguments may introduce very small divisors leading to spurious terms. In that case it is wise to purge the series of divisors which are not acceptable to a given precision (terms associated with very long periods of several millennia). As an example let us mention the following combination of arguments, which is very critical: $2 D-2 F+2 \Omega-2 L_{\mathrm{E}}-2 p_{\mathrm{A}}$. Using the frequencies of nutation theory the corresponding period is 200 million years! Obviously if such a linear combination appears in series manipulations it produces a "numerical noise" without any physical meaning.

Taking the derivative reduces by one order in $t$ the secular developments in (A.1). In the computation of $s(t)$ given by (6) we carry out a differentiation which is followed by an integration. Even though the integration formally raises the development by one order in $t$ the highest-order terms have already been lost and the final order is lowered by one.

One other difficulty to which the user should pay careful attention is the value of the iteration counter in an iterative process. As an example the inverse $1 / S$ of a series $S$ can be obtained by a Newton's Method starting from an approximate value of the inverse $(1 / S)_{0}$. One has to make sure that the convergence of the process after several iterations is guaranteed to the precision $\epsilon$. A key feature of GREGOIRE is its speed, which allows numerical tests to be carried out in order to validate the accuracy of the results.

\section{Appendix B: Numerical values of the basic quantities}

The expressions for the quantities $X, Y$ and $s$ are derived from expressions for the classical precession and nutation quantities based on the IAU 2000A precession-nutation model.
Table B.1. Phase and frequency of the luni-solar, planetary and precession arguments used in Table 1.

\begin{tabular}{lcr}
\hline \hline ARGUMENT & $\begin{array}{c}\text { PHASE } \\
\text { radian }\end{array}$ & \multicolumn{1}{c}{$\begin{array}{c}\text { FREQUENCY } \\
\text { radian/century }\end{array}$} \\
\hline$l$ & 2.3555557435 & 8328.6914257191 \\
$l^{\prime}$ & 6.2400601269 & 628.3019551714 \\
$F$ & 1.6279050815 & 8433.4661569164 \\
$D$ & 5.1984665887 & 7771.3771455937 \\
$\Omega$ & 2.1824391966 & -33.7570459536 \\
$L_{\mathrm{Me}}$ & 4.4026088420 & 2608.7903141574 \\
$L_{\mathrm{Ve}}$ & 3.1761466970 & 1021.3285546211 \\
$L_{\mathrm{E}}$ & 1.7534703140 & 628.3075849991 \\
$L_{\mathrm{Ma}}$ & 6.2034809130 & 334.0612426700 \\
$L_{\mathrm{J}}$ & 0.5995464970 & 52.9690962641 \\
$L_{\mathrm{Sa}}$ & 0.8740167570 & 21.3299104960 \\
$L_{\mathrm{U}}$ & 5.4812938720 & 7.4781598567 \\
$L_{\mathrm{Ne}}$ & 5.3118862870 & 3.8133035638 \\
\hline
\end{tabular}

The precession expressions of Lieske et al. (1977) are:

$$
\begin{aligned}
\psi_{A} & =5038.7784^{\prime \prime} \times t-1.07259^{\prime \prime} \times t^{2}-0.001147^{\prime \prime} \times t^{3} \\
\omega_{A} & =\epsilon_{0}+0.05127^{\prime \prime} \times t^{2}-0.007726^{\prime \prime} \times t^{3} \\
\epsilon_{A} & =\epsilon_{0}-46.8150^{\prime \prime} \times t-0.00059^{\prime \prime} \times t^{2}+0.001813^{\prime \prime} \times t^{3} \\
\chi_{A} & =10.5526^{\prime \prime} \times t-2.38064^{\prime \prime} \times t^{2}-0.001125^{\prime \prime} \times t^{3}
\end{aligned}
$$

with $\epsilon_{0}=84381.448^{\prime \prime}$.

The phases and frequencies of the luni-solar and planetary arguments of the IAU 2000 nutation provided in Table B.1 use the fundamental arguments of nutation as provided in Simon et al. (1994) (Tables 3.4 (b.3), 3.5 (b) and 5.8) based on IERS 1992 constants and Williams et al. (1991) for precession):

$$
\begin{aligned}
l= & 134.96340251+1717915923.2178 \times t+31.8792 \times t^{2} \\
& +0.051635 \times t^{3}-0.00024470 \times t^{4} \\
l^{\prime}= & 357.52910918+129596581.0481 \times t-0.5532 \times t^{2} \\
& +0.000136 \times t^{3}-0.00001149 \times t^{4} \\
F= & 93.27209062+1739527262.8478 \times t-12.7512 \times t^{2} \\
& -0.001037 \times t^{3}+0.00000417 \times t^{4} \\
D= & 297.85019547+1602961601.2090 \times t-6.3706 \times t^{2} \\
& +0.006593 \times t^{3}-0.00003169 \times t^{4} \\
\Omega= & 125.04455501-6962890.5431 \times t+7.4722 \times t^{2} \\
& +0.007702 \times t^{3}-0.00005939 \times t^{4}
\end{aligned}
$$

where the phases are in degrees, the time coefficients are in arcseconds and $t$ is measured in Julian centuries of TDB.

The expression used for $p_{\mathrm{A}}$ is in the same units:

$$
p_{\mathrm{A}}=0.0243817500 \times t+0.00000538691 \times t^{2} \text {. }
$$

\section{Appendix C: References for the numerical developments}

Table C.1 provides the location of the numerical expressions used in this paper. It also indicates the places where the definitive and full forms of the numerical expressions described in this paper are available. 
Table C.1. Location of the numerical expressions used, or provided, in this paper.

\begin{tabular}{cc}
\hline \hline QUANTITY & LOCATION \\
\hline Frame bias $\mathrm{d} \psi_{0}, \mathrm{~d} \epsilon_{0}$ & \\
Equinox offset $\mathrm{d} \alpha_{0}$ & MHB 2000 \\
Precession rate corrections $\mathrm{d} \psi_{\mathrm{A}}, \mathrm{d} \epsilon_{\mathrm{A}}$ & Chapront et al. 2002 \\
IAU 2000A luni-solar and planetary nutation & MHB 2000 \\
\hline 2) Numerical expressions provided in the paper & MHB 2000 \\
Frame bias $\xi_{0}, \eta_{0}$ & \\
TRS to CRS matrix and hence CIP X,Y & This paper \\
$s+X Y / 2$ series & This paper \\
$X, Y$ series & IERS Conventions $(*)$ \\
\hline
\end{tabular}

* Tables for the series for $X, Y$ and $s+X Y / 2$ are available only in electronic form, at the CDS via anonymous ftp to cdsarc.u-strasbg.fr (130.79.128.5) or via http://cdsweb.u-strasbg.fr/cgi-bin/qcat?J/A+A/400/1145 and at http://maia.usno.navy.mil/ch5tables.html

\section{References}

Aoki, S., \& Kinoshita, H. 1983, Celest. Mech., 29, 335 Atkinson, R. d'E., \& Sadler, D. H. 1951, MNRAS, 111, 619

Capitaine, N. 1990, Celest. Mech. Dyn. Astr., 48, 127

Capitaine, N., Guinot, B., \& Souchay, J. 1986, Celest. Mech., 39, 283

Capitaine, N., Guinot, B., \& McCarthy, D. D. 2000, A\&A, 355, 398

Capitaine, N., Wallace, P. T., McCarthy, D. D. 2003, A\&A, submitted
Chapront, J., Chapront-Touzé M., \& Francou, G. 2002, A\&A, 387, 700

Fukushima, T. 1991, A\&A, 244, L11

Guinot, B. 1979, Basic Problems in the Kinematics of the Rotation of the Earth, in Time and the Earth's Rotation, ed. D. D. McCarthy, \& J. D. Pilkington (D. Reidel Publishing Company), pp. 7-18

Herring, T., Mathews, P. M., Buffett, B. A. 2002, J. Geophys. Res., 107, B4, 10.1029/2001JB000390

IERS Conventions 2000, http://maia.usno.navy.mil/ conv2000.html/icc5.ps, draft

Lieske, J. H., Lederle, T., Fricke, W., \& Morando, B. 1977, A\&A, 58,1

Mathews, P. M., Herring, T. A., \& Buffett, B. A. 2002, J. Geophys. Res., 107, B4, 10.1029/2001JB000165

McCarthy, D. D., \& Capitaine, N. 2002, in 2002, in IERS Technical Note No. 29, ed. N. Capitaine et al. (Verlag des Bundesamts für Kartographie und Geodäsie, Frankfurt am main), 85

Newcomb, S. 1895, in Astronomical Papers for the American Ephemeris and Nautical Almanac, AP, Vol. 5, Part IV-3

Seidelmann, P. K. 1982, Celest. Mech., 27, 79

Simon, J. L., Bretagnon, P., Chapront, J., et al. 1994, A\&A, 282, 663

Souchay, J., Loysel, B., Kinoshita, H., \& Folgueira, M. 1999, A\&AS, 135,111

Standish, E. M. 1981, A\&A, 101, L17.

Wahr, J. M. 1981, Geoph. J. R. Astr. Soc., 64, 705.

Wallace, P. T. 2002, in IERS Technical Note No. 29, ed. N. Capitaine et al. (Verlag des Bundesamts für Kartographie und Geodäsie, Frankfurt am main), 65

Williams, 1994, AJ, 108(2), 711

Williams, J. G., Newhall, X. X., \& Dickey, J. O. 1991, AJ, 241, L9 